\title{
From MASK Knowledge Management methodology to learning activities described with IMS - LD
}

\author{
Djilali Benmahamed $^{1,2}$, Pierre Tchounikine ${ }^{2}$,Jean-Louis Ermine ${ }^{1}$ \\ 1 : Laboratoire d'Informatique de l'Université du Maine - CNRS FRE 2730
}

Avenue Laennec, 72085 Le Mans cedex 9 - France

\{djilali.benmahamed, pierre.tchounikine\}@lium.univ-lemans.fr

2 : Institut National des Télécommunications, Département Systèmes d'Information

9, rue Charles Fourier, 91011 Evry Cedex - France

jean-louis.ermine@int-evry.fr

in Professional Knowledge Management, Editors: Klaus-Dieter Althoff, Andreas Dengel, Ralph Bergmann, Markus Nick, Thomas Roth-Berghofer, ISBN: 3-54030465-7, pp. 165 - 175, 2005

\begin{abstract}
In this paper we present how knowledge capitalized using the Knowledge Management Mask methodology can be used to design E-learning activities by matching Mask models and the concepts proposed by the IMSLearning Design modelling language. Our study consists in highlighting the elearning aspects encapsulated in these MASK models carried out around a domain of activity, via a writing these elements in the description language IMS - Learning Design, in a preoccupation of reusability and reengineering.
\end{abstract}

\section{Introduction}

The general context of the work is the integration of Knowledge Management principles and methodologies and E-Learning requirements. More precisely, we are interested in the construction of learning activities from Knowledge Management systems. Learning activities are activities designed to make learners/employees achieve a given set of actions that will help them internalize knowledge. This follows the pedagogic constructivist approach that promotes "learning by doing" rather than just reading documents. Let us consider an organization that uses a given project management methodology and has to train its employees to this method. Presenting documents that describe the method (i.e., inert e-learning material) is necessary, but not sufficient. It can be powerfully completed by a learning activity that consists in proposing to a group of $n$ employees to achieve a project following the methodology that they are supposed to learn, using E-Communication (Mail, Forum, Collaborative tools, etc.) to achieve this collaborative e-learning activity. 
Building such a learning activity requires first identifying the scenarios that will be proposed to the trainees, the different tasks to perform, the different roles to be distributed, etc. and then modelling these different aspects. When the knowledge that is to be acquired is part of the company Knowledge Management system, it appears natural to build the learning activities scenarios from the data stored in the Knowledge Management system.

In this paper, we present the way proposed to achieve such a process and, more precisely, the way proposed to construct learning scenarios from the Mask Knowledge Management methodology and to represent them using the IMS-Learning Design language. The paper shows the way that the educational scripts and training units can prove to be applicable to the objective of sharing and appropriation of knowledge capitalized within the MASK models. Also, it justifies the necessity of a rewrite step of the MASK toward educational engineering modeling norms, in particular the IMS - Learning Design language.

The second section of this paper briefly explains the Mask Knowledge Management methodology and the IMS-LD standard. We then explain the matching between the different Mask models and the different components of an IMS-LD scenario. In order to illustrate the process we take examples from the construction of a project management scenario. This example was used as a full-scale theoretical example (the KM model description is approximately of 30 A4 pages, however this was not processed in a company as we are only at the first steps of the methodology development).

\section{Mask, a method for knowledge capitalization}

Mask is an evolution of the MKSM method [1], [3] and [4]. It is now a robust, validated and operational method. It takes its origins in cognitive-based knowledge engineering approaches in which problem solving methods are represented under several aspects: classification of concepts, relations between concepts, prescriptive actions and behaviour laws [4]. Mask proposes seven models to help experts and knowledge engineers structure knowledge under systemic, ergo-cognitive, psychocognitive, historical and evolution analyses (see references for details): knowledge patrimony model, domain model, activity model, historical model, concept model, task model and temporal line model.

Mask method allows, through various models describing various points of view, to study in-depth the experts' knowledge and their systems of values at different levels of granularity. This facilitates its use for dividing, decentralizing, learning and adapting this knowledge and describing the company's activities. One of the benefits is the ease in updating the model, according to the evolution of knowledge [7]. This allows a better description and put in practice and thus reduces differences between documentation and reality. 


\section{IMS - Learning Design}

Learning Design aims at an evolution of e-learning by capturing the "process" of education rather than simply content. By describing sequences of collaborative learning activities, Learning Design offers a new approach to re-use in e-learning [2]. Learning Design has emerged as one of the most significant recent developments in elearning. From a standards/specifications perspective, IMS Global Learning Consortium has recently released the IMS Learning Design specification [5], based on the work of the Open University of the Netherlands (OUNL) on "Educational Modelling Language" [6], a notational language to describe a "meta-model" of instructional design. The OUNL coordinates an international EML/IMS Learning Design implementation group known as the Valkenburg group (2003), and OUNL has recently stated its intention to no longer continue developing EML, but instead focuses its energies on the new IMS Learning Design specification [8].

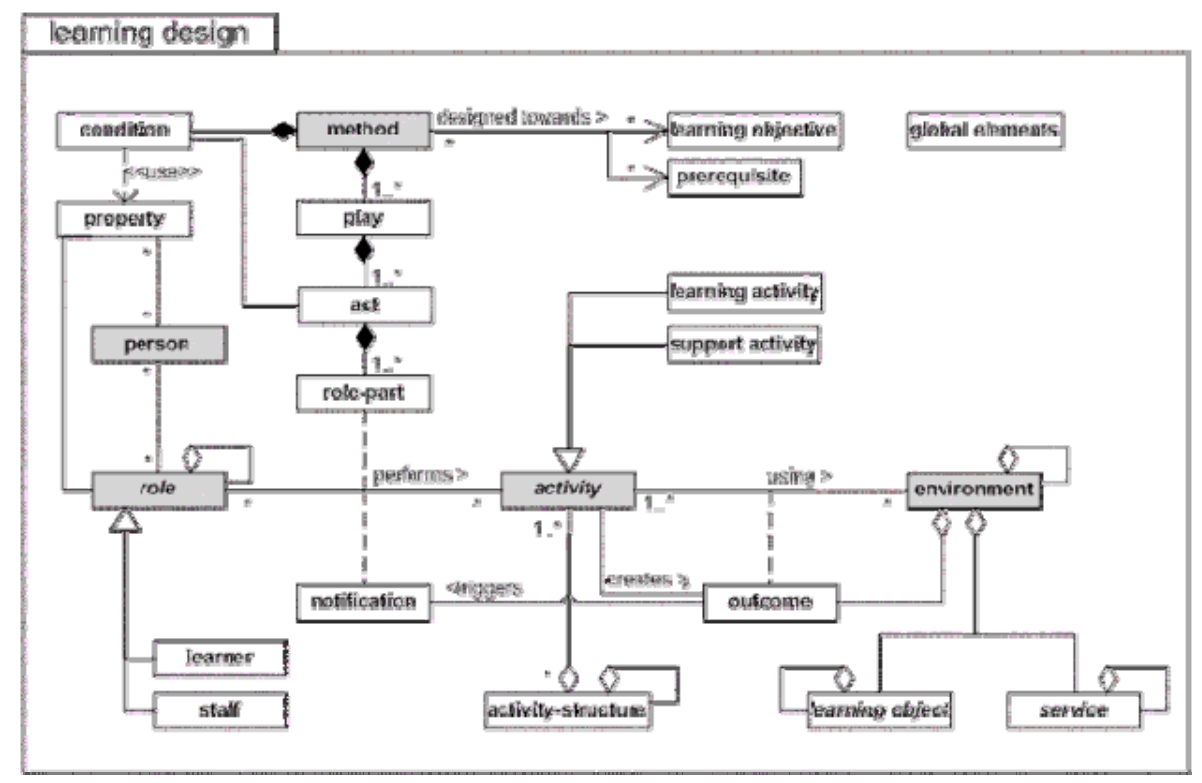

Fig. 1. Conceptual model of the overall Learning Design structure [5]

Three levels of representation suggested by IMS - Learning Design allow the specification and implementation of a great variety of e-learning teaching contents [5]. Level A specifies a time ordered series of activities to be performed by learners and teachers (role), within the context of an environment consisting of learning objects or services. Analysis of existing design approaches revealed that this was the common model behind all the different behaviorist, cognitive and (social) constructivist approaches to learning and instruction. For more advanced learning purposes, properties, conditions and notifications are required. This corresponds to Levels B and C. Properties, specified at Level B, are needed to store information 
about a person or a group of persons (role) e.g., for a student, its progress. Conditions, also part of Level B, constrain the evolution of the didactic scenario. They are set in response to specific circumstances, preferences or characteristics of specific learners (e.g., prior knowledge). Notifications, specified at Level $\mathrm{C}$ in addition to the properties and conditions of Level B are mechanisms to trigger new activities, based on an event during the learning process (e.g., the teacher is triggered to answer a question when a question of a student occurs or the teacher should grade a report once it has been submitted). In this paper we will limit ourselves to the "A" level, i.e., general design of scenario as time ordered activities.

\section{Matching Mask / IMS - Learning Design}

MASK models do not allow an efficient appropriation of the knowledge modelled by workers within the organization. However, one of the major objectives of such a capitalization and modelling remain that to share and re-use this knowledge. The simple access can not ensure an approval and a re-use of this knowledge. This modelling must thus meet a need more oriented learning.

Indeed, so that knowledge is re-used, it necessary that it is understood by the worker i.e. is integrated into its experiments and knowledge base and constantly mobilized in the action [10]. This approval and this reuse wished are therefore possible via a learning process, during which we show to the learner different domain difficulties and let's bring him the possible solutions that answer to these situations problems of operational order.

The development of a framework that supports pedagogical diversity and innovation, while promoting the exchange and interoperability of e-learning materials, is one of the key challenges in the e-learning industry today. The IMS Learning Design allows the elements and structure description of any unit of learning, including resources, instructions for learning activities, templates for structured interactions, conceptual models (e.g., problem-based learning), learning goals, objectives and outcomes and finally assessment tools and strategies [5].

The idea is to exploit the different concepts and aspects that contain the MASK models to extract and to structure the content of this learning. It justifies a step of MASK models rewrite toward modelling norms descended of the educational engineering, in particular the IMS-Learning Design language.

In the beginning, we tried to achieve this matching using the patrimony model. it was, for us, a starting point that permits a global vision described by the general phenomena, basis of the profession knowledge to distribute. The advantage is the faithful transcription of this global vision. The continuation was the deepening of every element representing a flux (of data, of information or cognitive). Once the definite global frame, deepening gives an indication on the granularity level of the learning scenario. We noted that such a gait denotes a starting a lot of general practitioner due to the generic level of the patrimony model that can generate several main activity models where each correlates to a well founded perception. 
Seen this first report, we experimented a matching from the activity model, where the main activity model is the starting point. The idea is to continue to describe the different steps of the scenario from the different correspondent's activities models and to continue while going until tasks models. Such a passage leads to a granularity level more and more refined. This gait proves to be interesting for the very detailed learning scenarios or that aim a training rather of initiation, thanks to the very detailed description level.

Seen this second report, we opted for a third way: to consider, since the departure, the granularity level of the learning scenario as defined by activities models. Patrimony, tasks and concepts models will remain elements to complete the different descriptions that ensue. The advantage of such a gait resides in the fact that we launch the matching with a maximum of precision and clarity. It's the choice that we kept and that we will retail in the following paragraphs, while starting with the general scenarios identification from the domain Model.

\section{Identifying general scenarios from the Domain Model}

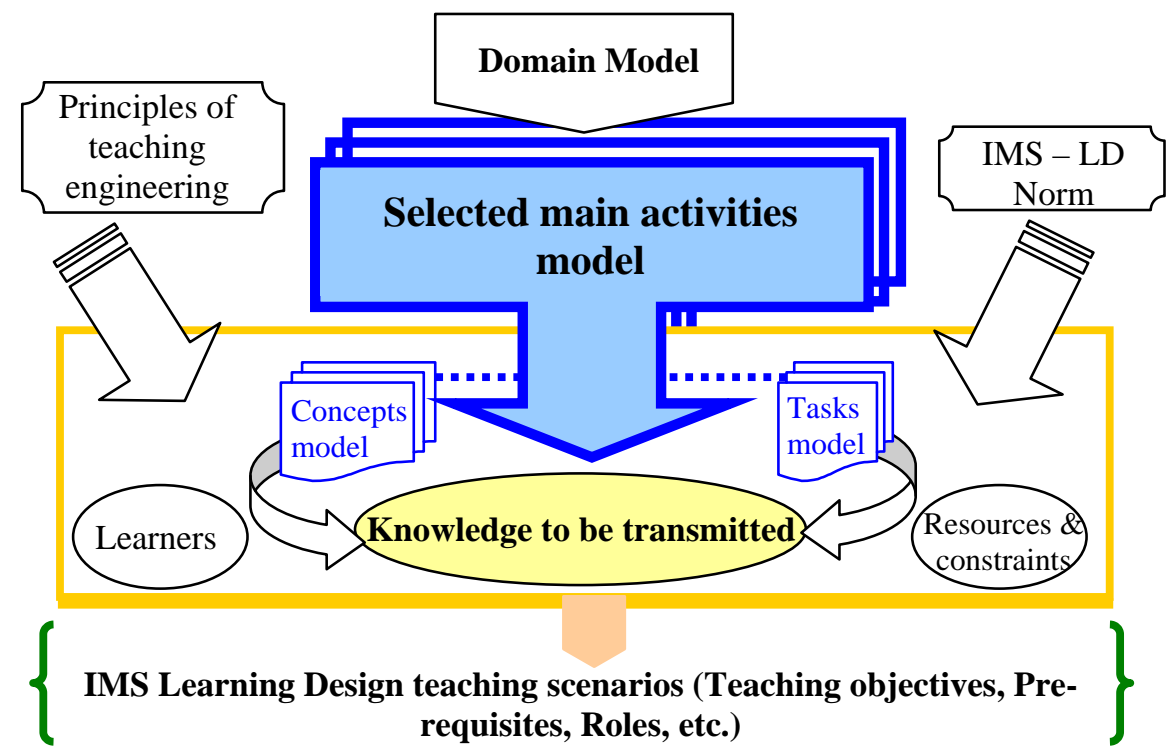

Fig. 2. Domain model generates various possibilities of scenarisation

MASK domain model proposes a vision sufficiently global of modeled knowledge that justifies its exploitation to identify the general scenario(s) of the learning activities. The continuation will be the deepening of each sub-element of this general model denoting a flow. This process emphasizes the principle based on the perception of a field like a recursive decomposition of phases and sub-phases. The idea is thus to describe the various headings of the teaching scenario by going through these phases. The general framework will be defined starting from the domain model and the 
succession of the decompositions will give an indication about the granularity level of the teaching scenarisation. So, a domain model can provide several scenarios corresponding to its different activities (Fig. 2). As an example, in the project management field, different scenarios can be identified corresponding to different general activities such as "definition of project", "team management", "dealing with the project resources" or in a more general manner "managing a project".

\section{Defining the scenarios from the main activities model}

The general scenario can be further detailed as follows:

- The patrimony model allows defining the different scenarios elements such as global prerequisites or the global teaching objectives the main activities model and the different activity steps (cf. Fig. 3).

- The main activities model allows making more precise the different activity steps by defining different characteristics such as the step number, the title and in particular the different actions to be scheduled (cf. Fig. 4).

- The different activity sub-models and their corresponding tasks models and concept models allow making more precise the different features of the learning activities such as the different roles, the teaching objectives or the intended production (cf. Fig. 5).

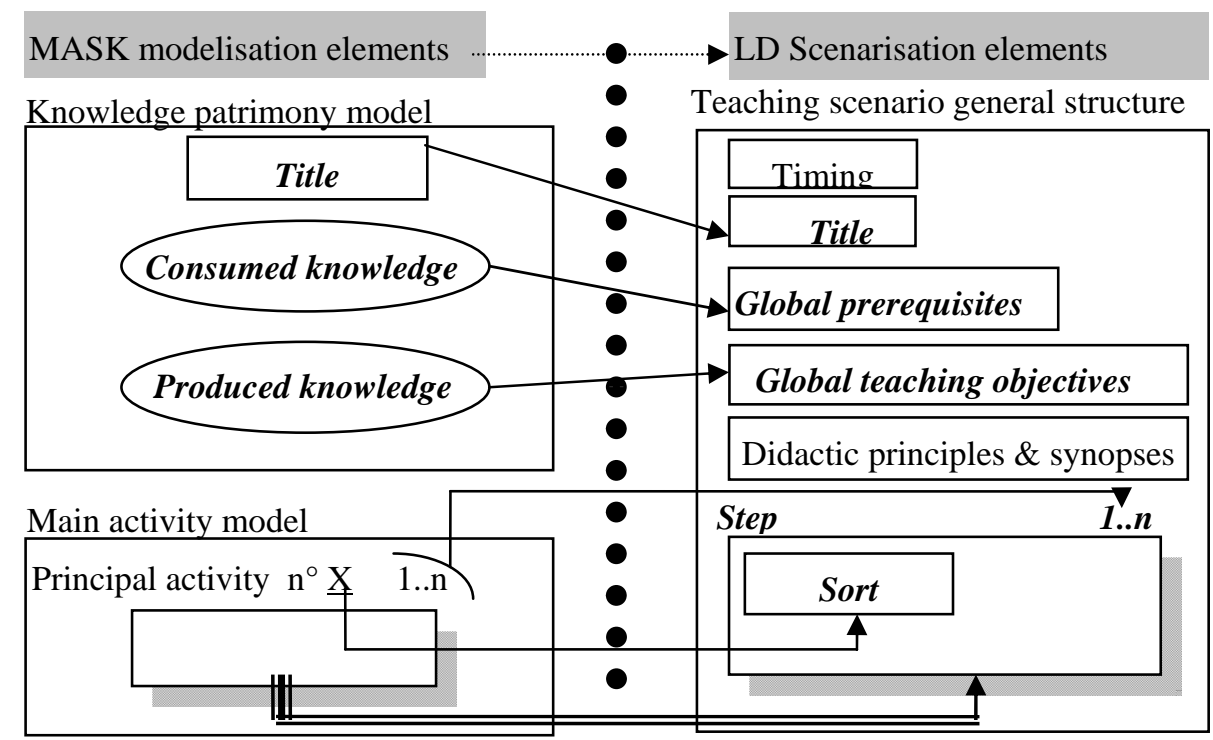

Fig. 3. Defining the scenario general structure 


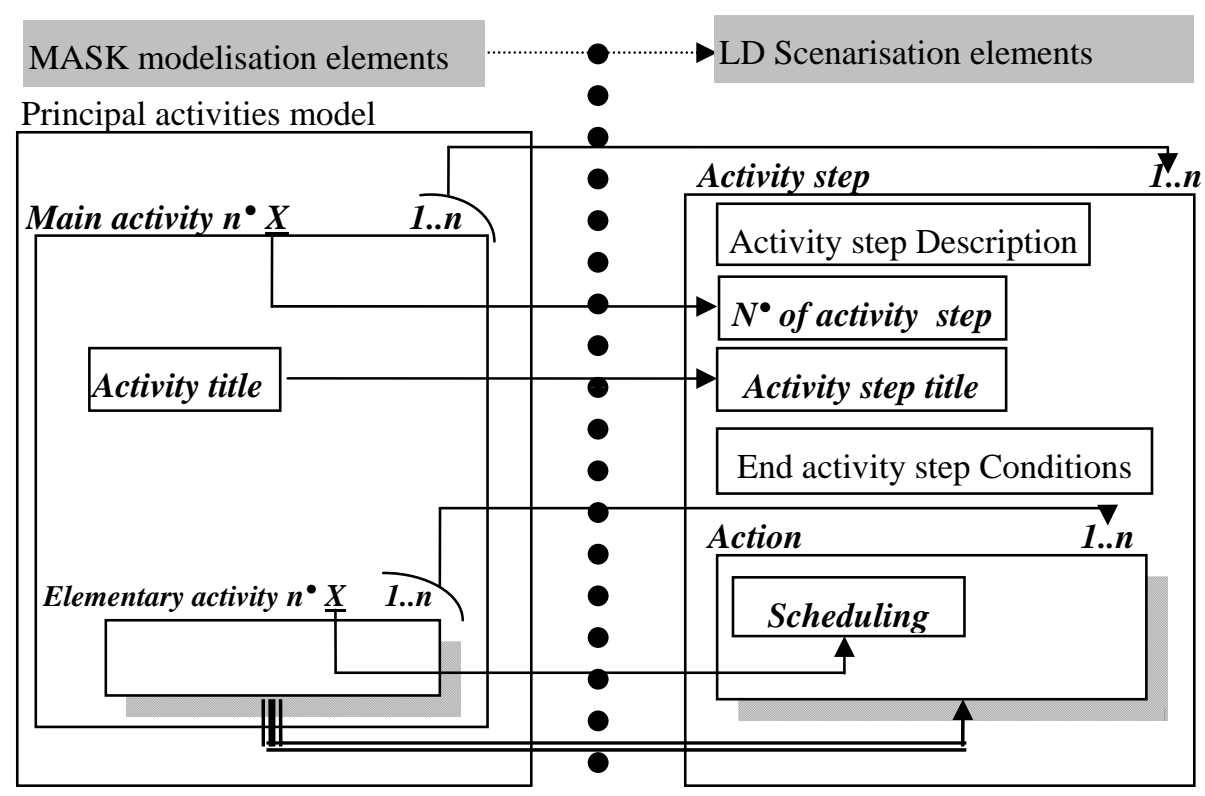

Fig. 4. Defining the different activity steps

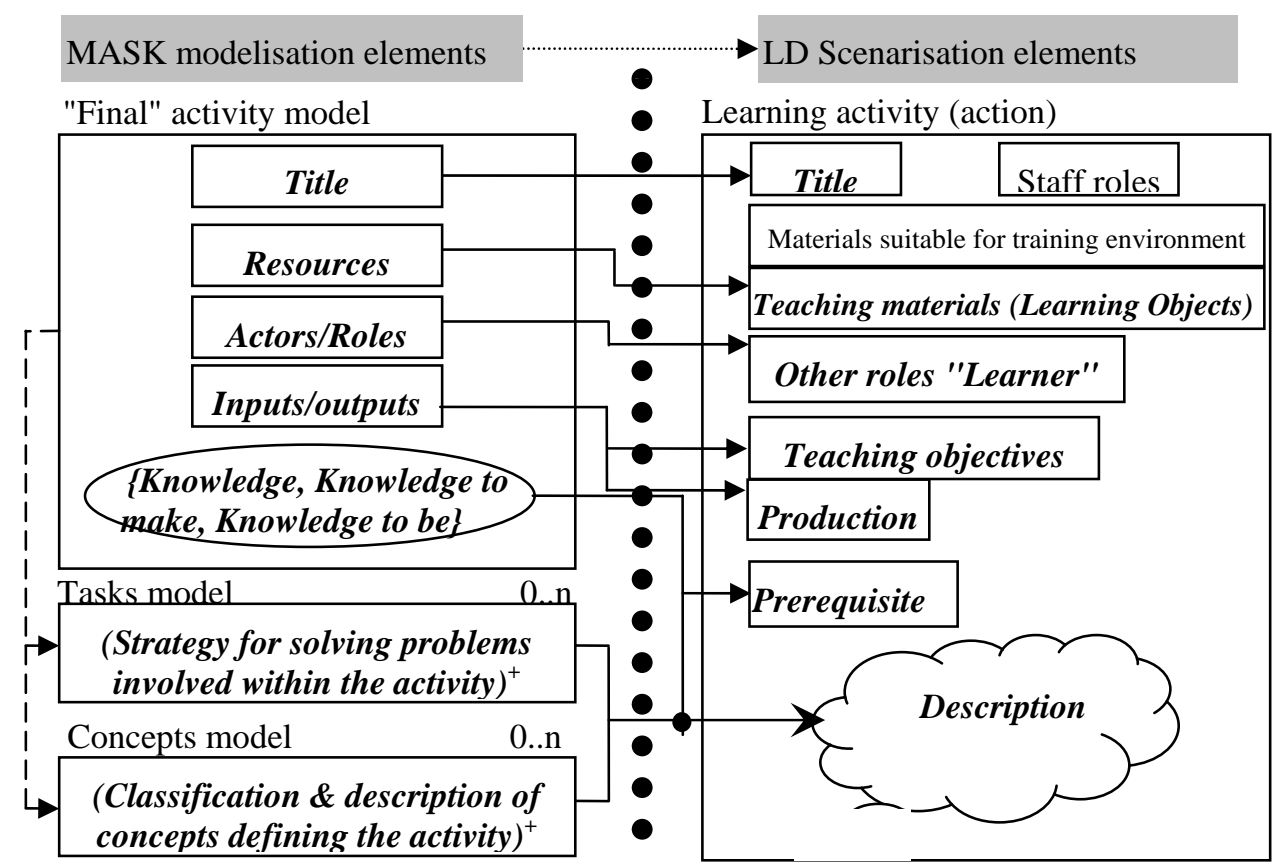

Fig. 5. Defining the details of the different activities 
If necessary, when the objective of the learning is to work out accurately a given procedure, the learning activity can be further detailed using the tasks model of the considered activity (the task model describes, in particular, the "expert" problem resolving strategy).

\section{Generating the IMS-LD description}

Table 1 presents an example of general learning scenario obtained following this process in the case of project management. This general scenario, described as a structured document, is based on 6 activity steps, each step being then refined into 24 activities. An IMS-LD description as an XML file can be generated from the standard description of this material (cf. fig. 6).

Table 1. A learning scenario

\begin{tabular}{|c|c|c|c|c|}
\hline \multicolumn{5}{|c|}{ Learning activity general structure for "project management" } \\
\hline \multicolumn{4}{|c|}{ Course title: Learning how to manage a project. } & Timing: $\mathrm{xxx}$ \\
\hline \multicolumn{5}{|c|}{$\begin{array}{l}\text { - developing high level competences in the project scenario definition, } \\
\text { dashboard construction, men management, } \\
\text { - ... }\end{array}$} \\
\hline \multicolumn{5}{|c|}{$\begin{array}{l}\text { Global prerequisites: fundamental knowledge on the project devices \& knowledge of } \\
\text { the project ecology ... }\end{array}$} \\
\hline \multicolumn{5}{|c|}{ 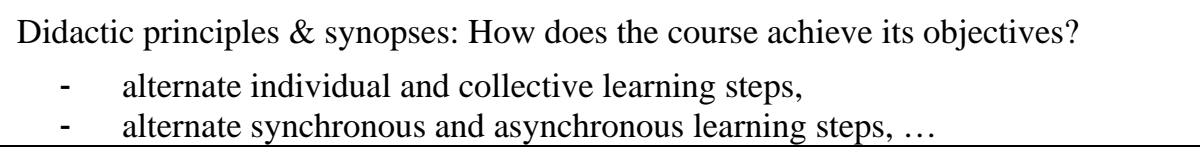 } \\
\hline \multicolumn{5}{|c|}{ Learning steps (Learning steps references and execution conditions) } \\
\hline Ref. & $\begin{array}{l}\text { Starting } \\
\text { with? }\end{array}$ & $\begin{array}{l}\text { Waiting the end } \\
\text { of learning step }\end{array}$ & Learning step title & \begin{tabular}{|c|}
$\begin{array}{c}\text { Next Learning } \\
\text { step }\end{array}$ \\
\end{tabular} \\
\hline 1 & Yes & 1 & $\begin{array}{l}\text { Preparation of the upstream } \\
\text { project }\end{array}$ & 2 \\
\hline 2 & No & 1 & Project beginning & $3,4,6$ \\
\hline$\ldots$ & $\ldots$ & $\ldots$ & $\ldots$ & $\ldots$ \\
\hline
\end{tabular}




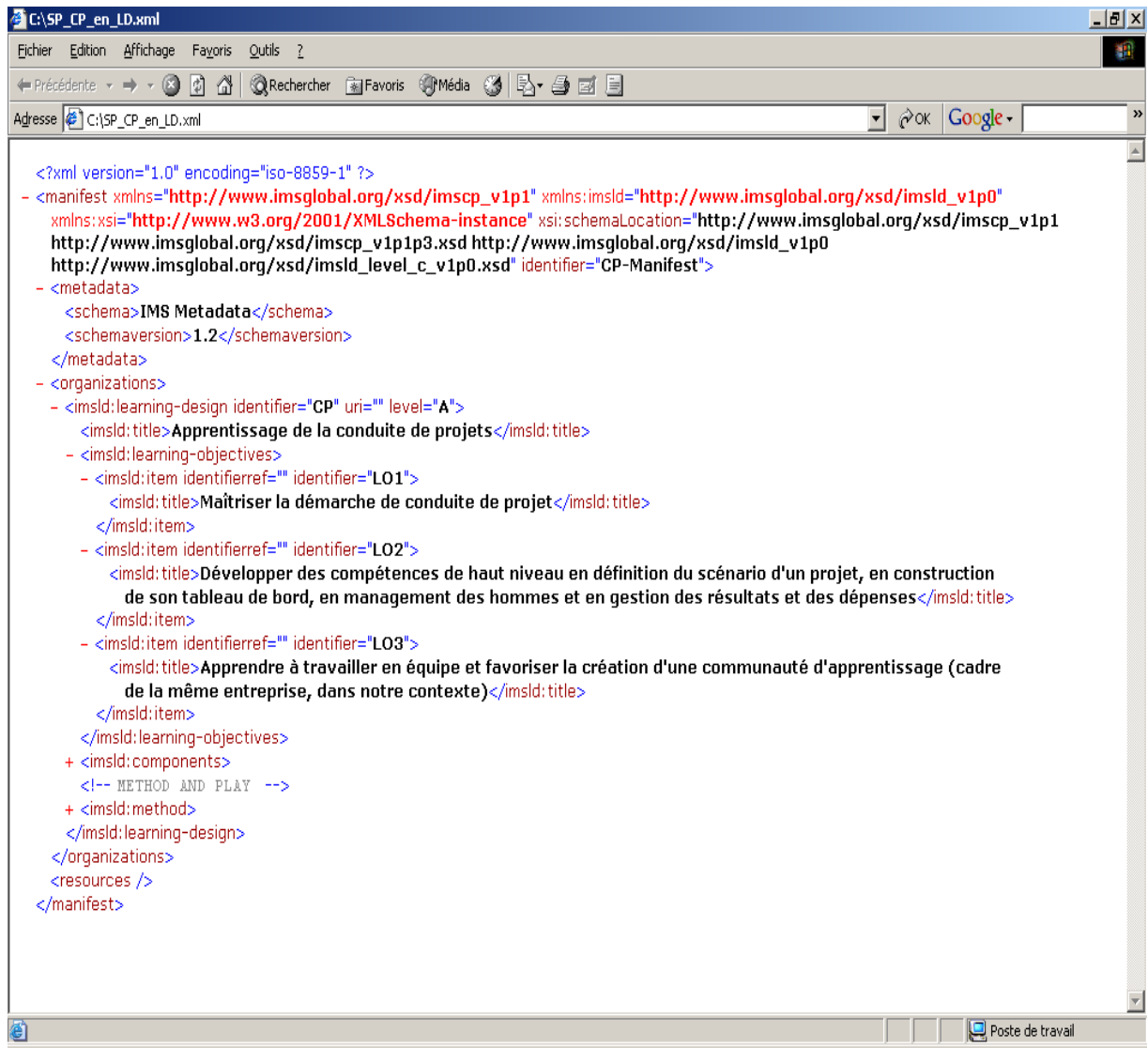

Fig. 6. IMS-LD General structure of the scenario for "project management" training

The result obtained was a teaching scenario which is characterized by:

- The existence of the main part of the e-learning aspects such as defined, distributed in various models MASK.

- Some missing elements: they acts primarily of the elements which are specific to the training process (staff roles, durations of the meetings, environment materials, etc.) and thus of the elements which were not taken into account at the time of modeling, nor thought by the expert during the interview of clarification.

- Difficulties noted for the definition of the level of granularity : MASK Models such as they are designed constitute "a block" of knowledge distributed on the various levels and models. In order to keep intact the direction of the knowledge-making, we had the constraint to adopt the same levels of granularity and decomposition. 
- Other constraints: elements in the teaching scenario cannot be directly inspired by the models MASK but which can be extracted well while choosing a combination from models. As an example, the description of the Learning activity requires elements of Knowledge, "Knowledge-to make" and "Knowledge-to be" corresponding model of the activity, descriptions of the tasks models and those of concepts models y referent.

\section{Conclusion}

Model-based approaches to Knowledge Management and E-learning present great convergences. Both have a finality of exchange and approval of knowledge. This can be used to study the passage from knowledge engineering models to e-learning scenarios. We have shown in this paper the way it can be done in the case of the Mask methodology and the IMS-LD standard.

The analysis of the approach we propose can be summarized as follows. The obtained scenarios cover the key knowledge that is proposed in the Mask models. However, some elements of the teaching scenario cannot be directly picked in a given Mask model but must be extracted from a combination of models. As an example, the description of learning activity requires elements such as "Knowledge-to make" and "Knowledge-to be" aspects that must be elicited from the activity model, the task models and the concept models. Moreover, some E-learning specific issues are not present in the Mask model and must be added through the process: staff roles, durations of the collaborative meetings, environment materials, etc. Finally, a difficult aspect of the matching is the definition of the learning activities level of granularity. Mask models constitute a "block" of knowledge distributed on the various levels and models. A learning activity generally focuses on a given issue at a given level of detail. Keeping coherent the levels of granularity of the two systems requires an accurate work that must be driven by pedagogic considerations.

Our definition of contents, design and scenarisation is intended to the actors of the field through an E-Learning platform and described in IMS - LD. That thus requires reflexions to reinforce the assets of such a passage and to answer the difficulties and/or lacks recorded at the time of the passage. For that, we propose for future developments:

- The expert can be called, during the interviews, to indicate some elements which it consider essential so that one learning can comparable its mode of reasoning and/or its way of resolution of problems

- To exploit the book of knowledge rather than the simple MASK models. The book of knowledge is, in fact, the "real" production of the method MASK and which includes the models. The book of knowledge has the advantage, compared to the models, to be content of complementary descriptions which answer the lacks that one noted theoretically and confirmed by our passage experimentation. 
Then, we believe that the development of methods that focus on constructing Elearning activities from $\mathrm{KM}$ systems is absolutely necessary to manage the complexity of E-learning issues in industrial companies. As an example, the Mask model of the "project management method" produced more than thirty models (plus their documentations). This cannot be managed "by hand". Although the process of building E-learning activities and curricula cannot be straightforward, approaches such as the one we propose guides and facilitates the process. Moreover, such an approach maintains the knowledge life cycle within the organization and allows reusability and reengineering thanks to IMS - Learning Design descriptions. We believe that knowledge engineering and teaching engineering issues models and systems can then progress towards interoperability.

\section{References}

1. Barthelmé F., Ermine J.L., Rosenthal-Sabroux C. An architecture for knowledge evolution in organisations, European Journal of Operational Research 109, 414-427 (1998)

2. Dalziel J., Implementing learning design: Then Learning Activity Management System (LAMS), Macquarie E-learning Centre of Excellence (MELCOE) - Macquarie University, Australia 2003.

3. Ermine J-L., Chaillot M., Bigeon P., Charreton B., Malavieille D. : MKSM, a method for knowledge management, Knowledge Management, Organization, Competence and Methodology, Advances in Knowledge Management Volume 1, Jos. F. Shreinemakers Ed., pp 288 - 302, Ergon, 1996

4. Ermine J-L: Les systèmes de connaissances, Edition Hermès, Paris, 2000

5. IMS Learning Design specification: http://www.imsglobal.org/learningdesign/index.cfm

6. Koper, R., From change to renewal: Educational technology foundations of electronic environments. EML website http://eml.ou.nl/eml-ou-nl.htm

7. Matta N., Ermine J.L., Aubertin G., Trivin J.Y., Knowledge Capitalization with a knowledge engineering approach: the Mask method, proceedings of IJCAI'2001 Workshop on Knowledge Management and Organizational Memory, August 2001.

8. Tattersall, C., EML and IMS Learning Design. Presentation for the Valkenburg Group, Vancouver, February 2003.

9. Tixier B., Rapport de recherche $n^{\circ}$ 01.9, Institut de recherche en informatique de Nantes, Septembre 2001.

10. Tounkara T., Ermine J-L., Matta N., L'approbation des connaissances avec MASK, In proceedings of Extraction et Gestion des Connaissances EGC'2002 (industrial session), Montpellier 2002. 